\title{
Galactic evolution of oxygen
}

\section{OH lines in 3D hydrodynamical model atmospheres}

\author{
J. I. González Hernández ${ }^{1,2,3}$, P. Bonifacio ${ }^{2,3,4}$, H.-G. Ludwig ${ }^{2,3,5}$, E. Caffau ${ }^{3}$, N. T. Behara ${ }^{2,3,6}$, and B. Freytag ${ }^{7,8}$ \\ 1 Dpto. de Astrofísica y Ciencias de la Atmósfera, Facultad de Ciencias Físicas, Universidad Complutense de Madrid, 28040 Madrid, \\ Spain \\ e-mail: jonay@astrax.fis.ucm.es \\ ${ }^{2}$ Cosmological Impact of the First STars (CIFIST) Marie Curie Excellence Team http://cifist. obspm. fr, France \\ ${ }^{3}$ GEPI, Observatoire de Paris, CNRS, Université Paris Diderot, Place Jules Janssen, 92190 Meudon, France \\ 4 Istituto Nazionale di Astrofisica - Osservatorio Astronomico di Trieste, via Tiepolo 11, 34143 Trieste, Italy \\ 5 Zentrum für Astronomie der Universität Heidelberg, Landessternwarte, Königstuhl 12, 69117 Heidelberg, Germany \\ ${ }^{6}$ Institut d'Astronomie et d'Astrophysique, Université Libre de Bruxelles, 1050 Bruxelles, Belgium \\ 7 CRAL,UMR 5574: CNRS, Université de Lyon, École Normale Supérieure de Lyon, 46 allée d'Italie, 69364 Lyon Cedex 7, France \\ ${ }^{8}$ Istituto Nazionale di Astrofisica - Osservatorio Astronomico di Capodimonte, via Moiariello 16, 80131 Napels, Italy
}

Received 10 March 2010 / Accepted 17 May 2010

\section{ABSTRACT}

\begin{abstract}
Context. Oxygen is the third most common element in the Universe. The measurement of oxygen lines in metal-poor unevolved stars, in particular near-UV OH lines, can provide invaluable information about the properties of the Early Galaxy.

Aims. Near-UV OH lines constitute an important tool to derive oxygen abundances in metal-poor dwarf stars. Therefore, it is important to correctly model the line formation of $\mathrm{OH}$ lines, especially in metal-poor stars, where 3D hydrodynamical models commonly predict cooler temperatures than plane-parallel hydrostatic models in the upper photosphere.

Methods. We have made use of a grid of 52 3D hydrodynamical model atmospheres for dwarf stars computed with the code $\mathrm{CO}^{5} \mathrm{BOLD}$, extracted from the more extended CIFIST grid. The 52 models cover the effective temperature range 5000-6500 K, the surface gravity range $3.5-4.5$ and the metallicity range $-3<[\mathrm{Fe} / \mathrm{H}]<0$.

Results. We determine 3D-LTE abundance corrections in all 52 3D models for several $\mathrm{OH}$ lines and Fe I lines of different excitation potentials. These 3D-LTE corrections are generally negative and reach values of roughly -1 dex (for the $\mathrm{OH} 3167$ with excitation potential of approximately $1 \mathrm{eV}$ ) for the higher temperatures and surface gravities.

Conclusions. We apply these 3D-LTE corrections to the individual $\mathrm{O}$ abundances derived from $\mathrm{OH}$ lines of a sample the metal-poor dwarf stars reported in Israelian et al. (1998, ApJ, 507, 805), Israelian et al. (2001, ApJ, 551, 833) and Boesgaard et al. (1999, AJ, 117, 492) by interpolating the stellar parameters of the dwarfs in the grid of 3D-LTE corrections. The new 3D-LTE [O/Fe] ratio still keeps a similar trend as the $1 \mathrm{D}-\mathrm{LTE}$, i.e., increasing towards lower $[\mathrm{Fe} / \mathrm{H}]$ values. We applied 1D-NLTE corrections to 3D Fe I abundances and still see an increasing $[\mathrm{O} / \mathrm{Fe}]$ ratio towards lower metallicites. However, the Galactic $[\mathrm{O} / \mathrm{Fe}]$ ratio must be revisited once 3D-NLTE corrections become available for $\mathrm{OH}$ and $\mathrm{Fe}$ lines for a grid of 3D hydrodynamical model atmospheres.
\end{abstract}

Key words. nuclear reactions, nucleosynthesis, abundances - stars: abundances - stars: Population II - Galaxy: halo Galaxy: evolution - line: formation

\section{Introduction}

The metal-poor stars of the Galactic halo provide the fossil record of the early Galaxy's composition. Dwarf halo stars are particularly relevant, because their atmospheres are not significantly altered by internal mixing and provide a unique tracer to constrain Galactic chemical evolutionary models. Oxygen is a key element in this scenario, because it is the most abundant element in stars after $\mathrm{H}$ and $\mathrm{He}$. It is produced in the interiors of massive stars by hydrostatic burning and its content is modified during the explosive nucleosynthesis in type II supernovae ( $\mathrm{SNe}$ ) and hypernovae $(\mathrm{HNe})$ and returned into the interstellar medium. On the other hand, iron is created by both type II and type I SN explosions. However, type I SNe progenitors have longer lifetimes, which is why the abundance ratio of $[\mathrm{O} / \mathrm{Fe} \mathrm{I}]$ can be used to constrain the chemical evolution of the Galaxy.

There have been numerous studies of the oxygen abundance in halo stars. Despite considerable observational and theoretical efforts, the trend of $[\mathrm{O} / \mathrm{Fe}]$ ratio $^{1}$ versus $[\mathrm{Fe} / \mathrm{H}]$ is still unclear. The analysis of the forbidden line OI $6300 \AA$ in giants shows a plateau with $[\mathrm{O} / \mathrm{Fe}] \sim 0.4-0.5$ in the metallicity range $-2.5<[\mathrm{Fe} / \mathrm{H}]<-1$ (Barbuy 1988) and $[\mathrm{O} / \mathrm{Fe}] \sim 0.7$ for -4 . $<$ $[\mathrm{Fe} / \mathrm{H}]<-2.5$ (Cayrel et al. 2004). A similar behaviour is seen for metal-poor subgiant stars in the range -3 . $<[\mathrm{Fe} / \mathrm{H}]<-1.5$ ([O/Fe] 0.4-0.5, García Pérez et al. 2006). However, García Pérez et al. (2006) already noted that by plotting all measurements from the [OI] line for dwarfs (Nissen et al. 2002), subgiants (García Pérez et al. 2006) and giants (Cayrel et al. 2004), the picture changes and an increasing trend $[\mathrm{O} / \mathrm{Fe}]$ towards lower metallicities clearly appears.

The near infrared (IR) triplet O I 7771-5 $\AA$ in metal-poor dwarfs and subgiants (Abia \& Rebolo 1989; Israelian et al. 2001; García Pérez et al. 2006) points towards increasing [O/Fe] values with decreasing $[\mathrm{Fe} / \mathrm{H}]$, although the $\mathrm{O}$ abundances derived

\footnotetext{
${ }^{1}[\mathrm{O} / \mathrm{Fe}]=\log [N(\mathrm{O}) / N(\mathrm{Fe})]_{\star}-\log [N(\mathrm{O}) / N(\mathrm{Fe})]_{\odot}$.
} 
from the near-IR triplet are typically $\sim 0.4-0.7$ higher than those derived from the forbidden O I line (Fulbright \& Johnson 2003). The $\mathrm{OH}$ A-X electronic lines in the near ultraviolet (UV) provide also higher $[\mathrm{O} / \mathrm{Fe}]$ ratios towards lower $[\mathrm{Fe} / \mathrm{H}]$ values in dwarf stars (Israelian et al. 1998; Boesgaard et al. 1999; Israelian et al. 2001; González Hernández et al. 2008). However, García Pérez et al. (2006) found a quasi-plateau of $[\mathrm{O} / \mathrm{Fe}]$ for subgiant stars in the range $-3 .<[\mathrm{Fe} / \mathrm{H}]<-1.5$, using $\mathrm{Fe}$ abundances determined from $\mathrm{Fe}$ II lines. The $[\mathrm{O} / \mathrm{Fe}]$ ratio shows a negative slope if one instead uses the Fe abundances estimated from $\mathrm{Fe}$ I lines, although with lower $[\mathrm{O} / \mathrm{Fe}]$ values than those determined for metal-poor dwarfs. It is advisable to use Fe I lines instead of Fe II lines to derive the $[\mathrm{O} / \mathrm{Fe}]$ ratio because of its similar sensitivity to the surface gravity.

These O-abundance indicators present different complications. The near-IR O I triplet is susceptible to non-local thermodynamical equilibrium (NLTE) effects (Kiselman 2001, and references therein), with abundance corrections below $0.2 \mathrm{dex}$, and is quite sensitive to the adopted $T_{\text {eff }}$. The [OI] is not sensitive to departures from LTE, but it is essentially undetectable in dwarfs with $[\mathrm{Fe} / \mathrm{H}] \lesssim-2$. The near-UV OH lines are strongly sensitive to the temperature structure and inhomoginities (Asplund \& García Pérez 2001; González Hernández et al. 2008). In addition, Fe I lines suffer from severe NLTE effects in metal-poor stars (see e.g. Thévenin \& Idiart 1999). We note that Shchukina et al. (2005) have performed NLTE computations for the metalpoor subgiant HD $140283([\mathrm{Fe} / \mathrm{H}] \sim-2.5)$ with a single snapshot of a 3D hydrodynamical simulation (Asplund et al. 1999), and found NLTE-LTE corrections of +0.9 and +0.4 for Fe I and Fe II lines, respectively.

Finally, the oxygen abundance in the Sun is still a matter of debate. We will adopt throughout this work the value of $\log \left[N(\mathrm{O}) / N(\mathrm{H}]_{\odot}=8.76\right.$, which was determined through 3-dimensional (3D) hydrodynamical models (Caffau et al. 2008).

We have used a subset of the CIFIST grid of 3D hydrodynamical model atmospheres (Ludwig et al. 2009) to investigate the 3D-LTE and 3D-NLTE $[\mathrm{O} / \mathrm{Fe}]$ and $[\mathrm{O} / \mathrm{H}]$ trends in metal-poor dwarf stars.

\section{3D hydrodynamical simulations}

The 3D hydrodynamical model atmospheres (see Ludwig et al. 2009, for further details) were computed with the code $\mathrm{CO}^{5}$ BOLD (Freytag et al. 2002; Wedemeyer et al. 2004). Each model consists of a representative set of snapshots sampling the temporal evolution of the photospheric flow. In Table 1 we provide a summary of the $3 \mathrm{D}$ models used in this paper. The evolutionary time scale and the spatial scale of the convective surface flows roughly scale inversely proportional to the surface gravity. Following this scaling behaviour, we tried to ensure that the duration of each model sequence corresponds to at least $3600 \mathrm{~s}$ of solar-equivalent time. Moreover, we also scaled the size of the computational domain leading to about the same total number of convective cells in each model.

The spatial resolution was typically $\left(n_{x} \times n_{y} \times n_{z}\right)=$ $(140,140,150)$, with a constant grid spacing in the (horizontal) $x$ - and $y$-directions and a variable spacing in $z$-direction. Our standard solar model - actually not part of the grid used here, but mentioned for reference - has a horizontal size of $5600 \mathrm{~km}$. The total height is $2250 \mathrm{~km}$, from $z=-1380 \mathrm{~km}$ below optical depth unity to $z=870 \mathrm{~km}$ above, located in the low chromosphere. The computational time step was typically $0.1-0.2 \mathrm{~s}$.
Table 1. Details of the $3 \mathrm{D}$ hydrodynamical $\mathrm{CO}^{5} \mathrm{BOLD}$ model atmospheres.

\begin{tabular}{|c|c|c|c|c|}
\hline $\begin{array}{l}\left\langle T_{\text {eff }}\right\rangle^{a} \\
{[\mathrm{~K}]}\end{array}$ & $\begin{array}{l}\log g \\
{[\mathrm{cgs}]}\end{array}$ & $\begin{array}{r}{[\mathrm{Fe} / \mathrm{H}]} \\
{[\mathrm{dex}]}\end{array}$ & $\begin{array}{r}\text { Time }^{b} \\
{[\mathrm{~s}]}\end{array}$ & Snapshots \\
\hline 4920 & 3.5 & 0 & 132000 & 19 \\
\hline 4930 & 3.5 & -1 & 420000 & 20 \\
\hline 4980 & 3.5 & -2 & 544000 & 20 \\
\hline 4980 & 3.5 & -3 & 464000 & 20 \\
\hline 4950 & 4.0 & 0 & 44000 & 20 \\
\hline 4990 & 4.0 & -1 & 235200 & 20 \\
\hline 4960 & 4.0 & -2 & 58401 & 20 \\
\hline 4990 & 4.0 & -3 & 54400 & 20 \\
\hline 4990 & 4.5 & 0 & 31200 & 20 \\
\hline 5060 & 4.5 & -1 & 56500 & 19 \\
\hline 5010 & 4.5 & -2 & 46500 & 19 \\
\hline 4990 & 4.5 & -3 & 29700 & 20 \\
\hline 5430 & 3.5 & 0 & 144000 & 18 \\
\hline 5480 & 3.5 & -1 & 106800 & 19 \\
\hline 5500 & 3.5 & -2 & 46800 & 20 \\
\hline 5540 & 3.5 & -3 & 201600 & 20 \\
\hline 5480 & 4.0 & 0 & 34800 & 20 \\
\hline 5530 & 4.0 & -1 & 95200 & 20 \\
\hline 5470 & 4.0 & -2 & 33800 & 20 \\
\hline 5480 & 4.0 & -3 & 60800 & 20 \\
\hline 5490 & 4.5 & 0 & 19000 & 20 \\
\hline 5470 & 4.5 & -1 & 48600 & 20 \\
\hline 5480 & 4.5 & -2 & 57000 & 20 \\
\hline 5490 & 4.5 & -3 & 54300 & 20 \\
\hline 5880 & 3.5 & 0 & 236000 & 20 \\
\hline 5890 & 3.5 & -1 & 200000 & 20 \\
\hline 5860 & 3.5 & -2 & 112000 & 20 \\
\hline 5870 & 3.5 & -3 & 112000 & 20 \\
\hline 5930 & 4.0 & 0 & 26400 & 18 \\
\hline 5850 & 4.0 & -1 & 27000 & 20 \\
\hline 5860 & 4.0 & -2 & 30000 & 20 \\
\hline 5850 & 4.0 & -3 & 30500 & 20 \\
\hline 5870 & 4.5 & 0 & 16900 & 19 \\
\hline 5920 & 4.5 & -1 & 7000 & 8 \\
\hline 5920 & 4.5 & -2 & 24500 & 18 \\
\hline 5920 & 4.5 & -3 & 9000 & 19 \\
\hline 6230 & 4.0 & 0 & 54000 & 20 \\
\hline 6260 & 4.0 & -1 & 43800 & 20 \\
\hline 6280 & 4.0 & -2 & 27600 & 16 \\
\hline 6270 & 4.0 & -3 & 46200 & 20 \\
\hline 6230 & 4.5 & 0 & 35400 & 20 \\
\hline 6240 & 4.5 & -1 & 25000 & 20 \\
\hline 6320 & 4.5 & -2 & 9100 & 19 \\
\hline 6270 & 4.5 & -3 & 22000 & 18 \\
\hline 6490 & 4.0 & 0 & 58200 & 20 \\
\hline 6500 & 4.0 & -1 & 57000 & 20 \\
\hline 6530 & 4.0 & -2 & 49200 & 20 \\
\hline 6410 & 4.0 & -3 & 56400 & 20 \\
\hline 6460 & 4.5 & 0 & 17400 & 20 \\
\hline 6460 & 4.5 & -1 & 36200 & 19 \\
\hline 6530 & 4.5 & -2 & 9100 & 19 \\
\hline 6450 & 4.5 & -3 & 2200 & 12 \\
\hline
\end{tabular}

Notes. ${ }^{(a)}$ Temporal average of the emergent $T_{\text {eff }} \cdot{ }^{(b)}$ Total time covered by the temporal evolution of the photospheric flow named as snapshots. 
J. I. González Hernández et al.: Oxygen versus Iron using 3D models

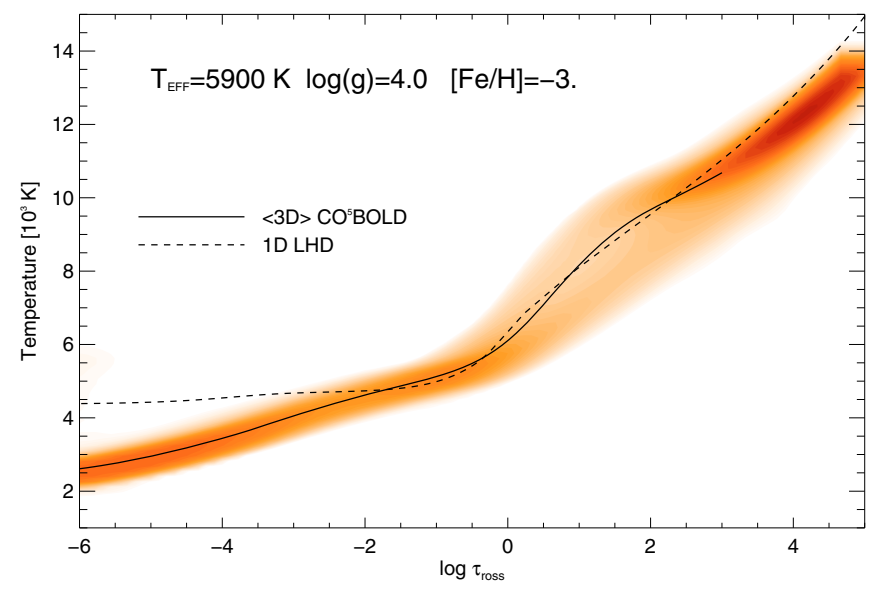

Fig. 1. 3D temperature structure of the 3D $\mathrm{CO}^{5} \mathrm{BOLD}$ atmospheric model, compared to the average temperature profile, $\langle 3 \mathrm{D}\rangle$, and the $1 \mathrm{D}_{\text {LHD }}$ model. Darker shades indicate more likely temperatures. The stellar parameters and metallicity of this 3D simulation are $T_{\text {eff }}[\mathrm{K}] / \log \left(g\left[\mathrm{~cm} \mathrm{~s}^{-2}\right]\right) /[\mathrm{Fe} / \mathrm{H}]=5850 / 4 /-3$.

The description of the radiative energy exchange is important for the resulting temperature structure of a model. Here we provide some details about the binning-scheme which we applied when modelling the wavelength dependence of the radiative transfer. The wavelength dependence is represented by 5 multi-group bins in solar metallicity models and 6 bins at sub-solar metallicities following the procedure originally laid out by Nordlund (1982) and subsequently refined by Ludwig (1992); Ludwig et al. (1994); Vögler et al. (2004). For test purposes we calculated a few models with 12 bins. Different from the original implementation of Nordlund (1982), the binaveraged opacities were calculated explicitely from the run of the monochromatic opacity within a particular bin. No scaling among the bins was assumed. The sorting into wavelength groups was done applying thresholds in logarithmic Rosseland optical depth $\{+\infty, 0.0,-1.5,-3.0,-4.5,-\infty\}$ for the 5-bin, and $\{+\infty, 0.1,0.0,-1.0,-2.0,-3.0,-\infty\}$ for the 6-bin schemes. For the 12 bins we used as thresholds $\{+\infty, 0.15,0.0$, $-0.75,-1.5,-2.25,-3.0,-3.75,-4.5,-\infty\}$; in addition, each of the first three continuum-like bins were split into 2 bins according to wavelength at 550,600, and $650 \mathrm{~nm}$. In all but one bin a switching between Rosseland and Planck averages was performed at a band-averaged Rosseland optical depth of 0.35 ; in the bin gathering the largest line opacities the Rosseland mean opacity was used throughout. The decisions about the number of bins, and sorting thresholds are motivated by comparing radiative fluxes and heating rates obtained by the binned opacities in comparison to high wavelength resolution. In comparison to the models of Asplund \& García Pérez (2001), who worked with Stein-Nordlund models (Stein \& Nordlund 1998) and used 4 opacity bins, our treatment of the wavelength dependence of the radiation field puts extra emphasis on the continuum-forming layers.

\subsection{D temperature structure}

In Figs. 1 and 2 we depict the temperature structure of two $3 \mathrm{D}$ hydrodynamical simulations for two different effective temperatures, 5850 and $6270 \mathrm{~K}$, and the same surface gravity, $\log \left(\mathrm{g} / \mathrm{cm} \mathrm{s}^{2}\right)=4$, and metallicity, $[\mathrm{Fe} / \mathrm{H}]=-3$. The main difference between these two $3 \mathrm{D}$ models is the temperature inhomogeneities which are larger in the hotter model. These strong

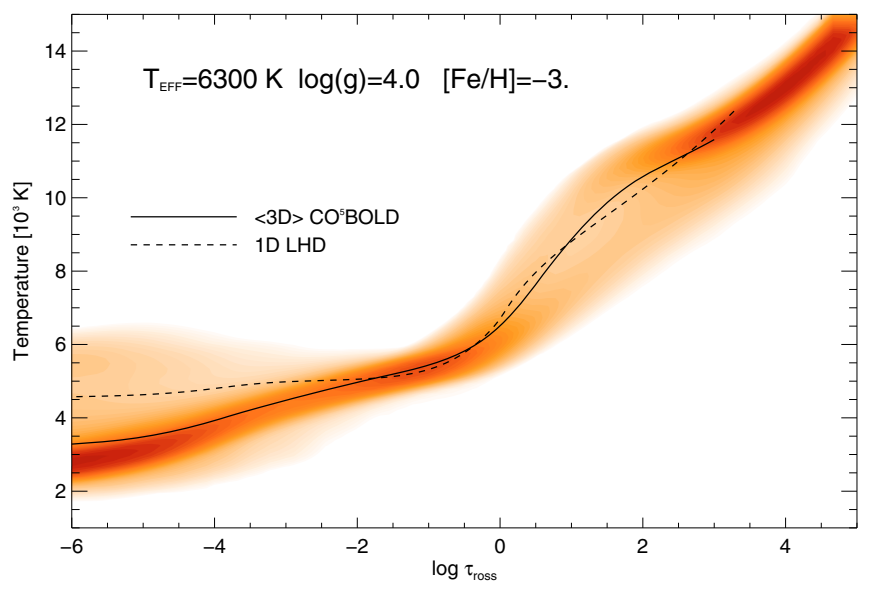

Fig. 2. Same as Fig. 1 but for the $3 \mathrm{D}$ model $T_{\text {eff }}[\mathrm{K}] / \log \left(g\left[\mathrm{~cm} \mathrm{~s}^{-2}\right]\right) /$ $[\mathrm{Fe} / \mathrm{H}]=6270 / 4 /-3$.

temperature fluctuations are typically present for effective temperatures hotter than $5900 \mathrm{~K}$ and surface gravities lower than 4 .

The 3D models displayed in Figs. 1 and 2 show an overcooling effect in the outer layers of the star with respect to 1D models. This is particularly relevant in all models with metallicities $[\mathrm{Fe} / \mathrm{H}]=-3$ and -2 . 3D models with metallicities $[\mathrm{Fe} / \mathrm{H}]=-1$ and 0 do not show a pronounced overcooling effect and the line formation is dominated by the temperature fluctuations. We note that the overcooling of the higher photospheric layers is the dominant 3D effect, but in the deep photosphere, at $\log \tau \sim-1$, $3 \mathrm{D}$ models are always slightly hotter than a corresponding hydrostatic 1D model in radiative-convective equilibrium.

The comparison of 3D versus 1D models depends on which particular 1D model is chosen. We compared each of our full 3D models to a corresponding (in $T_{\text {eff }}, \log g$, and metallicity) standard hydrostatic 1D model atmosphere (hereafter denoted as $\left.1 D_{\text {LHD }}\right)$ and a 1D model obtained from the temporal and horizontal average of the 3D structure over surfaces of equal (Rosseland) optical depth (hereafter denoted as $\langle 3 \mathrm{D}\rangle$ ). The $1 \mathrm{D}_{\mathrm{LHD}}$ model is calculated with a 1D atmosphere code called LHD. It assumes plane-parallel geometry and employs the same micro-physics (equation-of-state, opacities) as $\mathrm{CO}^{5} \mathrm{BOLD}$. Convection is described by mixing-length theory. See Caffau et al. (2007) for further details. Note that the $1 \mathrm{D}_{\text {LHD }}$ model depends on the choice of the mixing-length parameter, the $\langle 3 \mathrm{D}\rangle$ model on details of the averaging procedure. The temperature of the $\langle 3 \mathrm{D}\rangle$ model is indeed obtained by averaging the fourth power of the 3D temperature field. The choice was motivated by the property that this kind of averaging largely preserves the radiative flux (Steffen et al. 1995).

We adopted a mixing-length parameter $\alpha=1$ in the LHD models. However, this parameter only affects lines that are formed at optical depth $\log \tau>-0.5$. As we will see in the next section, the $\mathrm{OH}$ lines in metal-poor 1D models form at optical depth $\log \tau<-1$.

We compute the curves of growth of the $\mathrm{OH}$ lines and Fe I lines, for both 1D and 3D models with the spectral synthesis code Linfor $3 \mathrm{D}^{2}$. It is necessary to assume a value of the microturbulence for the spectral synthesis of the 1D models. We have adopted a $\xi_{\mathrm{t}}=1 \mathrm{~km} \mathrm{~s}^{-1}$.

\footnotetext{
${ }^{2}$ More information on Linfor3D can be found in the following link: http://www.aip.de/ mst/Linfor3D/linfor_3D_manual.pdf
} 


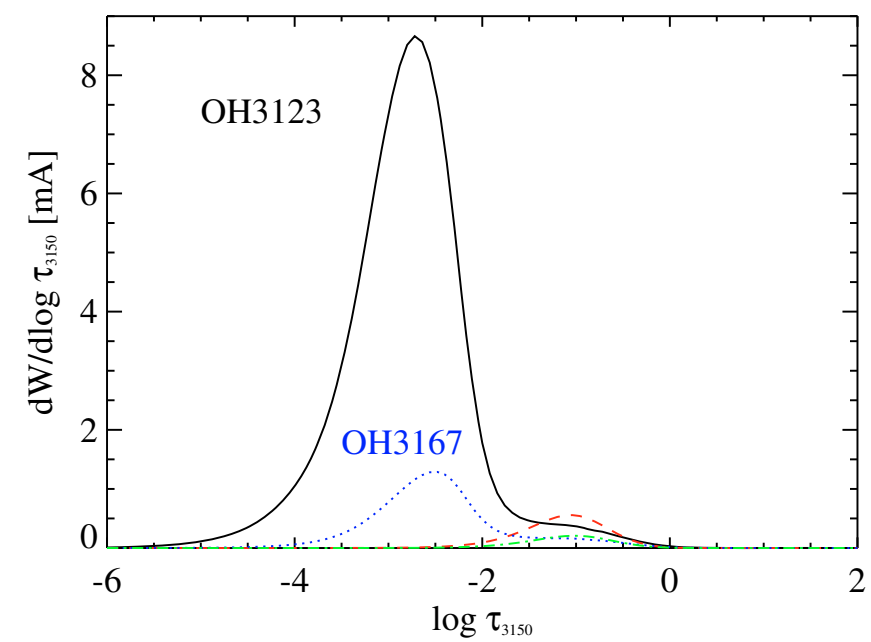

Fig. 3. Contribution functions to the equivalent width of the $\mathrm{OH}$ $3123 \AA$ line computed with $\mathrm{CO}^{5}$ BOLD3D model (solid line) and $1 \mathrm{D}_{\mathrm{LHD}}$ model (dashed line). The same is displayed for the $\mathrm{OH}$ $3167 \AA$ line (3D model, dotted line, and 1D $\mathrm{D}_{\mathrm{LHD}}$ model, dashed-dotted line). The stellar parameters and metallicity of this simulation are $T_{\text {eff }}[\mathrm{K}] / \log \left(g\left[\mathrm{~cm} \mathrm{~s}^{-2}\right]\right) /[\mathrm{Fe} / \mathrm{H}]=6270 / 4 /-3$.

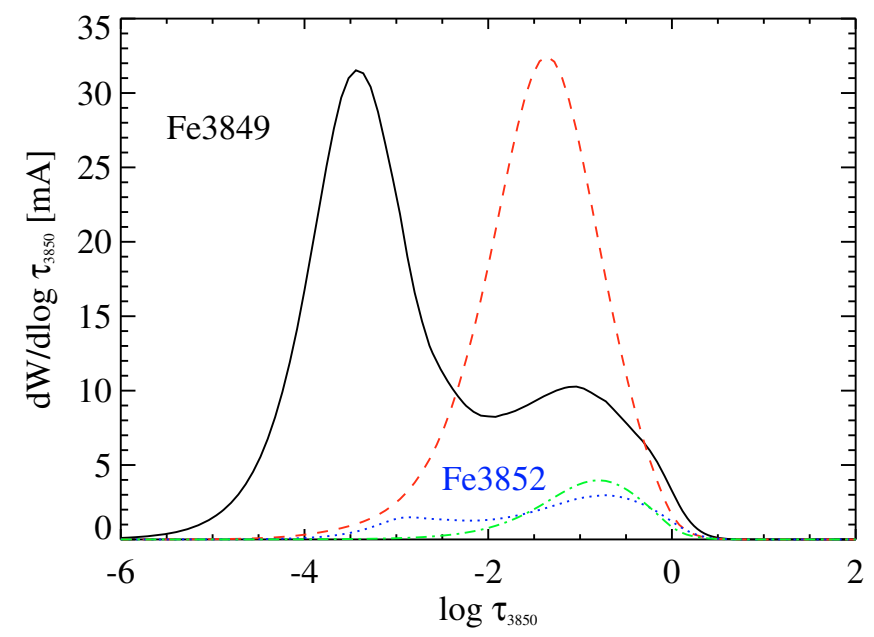

Fig. 4. Same as Fig. 3 but for the Fe I $3849 \AA$ And $3852 \AA$ lines.

\subsection{Line formation}

In Fig. 3 we display the contribution functions (CFs) to the equivalent width $(E W)$ of the disc centre of the $\mathrm{OH}$ lines at $3123 \AA$ and $3167 \AA$ according to the $\mathrm{CO}^{5} \mathrm{BOLD} 3 \mathrm{D}$ model with given stellar parameters and metallicity $T_{\text {eff }}[\mathrm{K}] / \log \left(g\left[\mathrm{~cm} \mathrm{~s}^{-2}\right]\right) /[\mathrm{Fe} / \mathrm{H}]=6270 / 4 /-3$. These two features have different excitation potentials $(\chi=0.2$ and $1.1 \mathrm{eV}$ for the OH $3123 \AA$ and $3167 \AA$ lines, respectively) and their contribution functions look similar in shape but different in strength. These molecular lines tend to form in the outer layers of the 3D model atmosphere, at $\log \tau$ between $\sim-2.8$ and $\sim-2.2$, where the $3 \mathrm{D}$ model is cooler than the $1 \mathrm{D}_{\mathrm{LHD}}$ model. Thus this line is significantly weaker in the $1 \mathrm{D}_{\mathrm{LHD}}$ model and forms in the inner layers of the star. Because of the sensitivity of $\mathrm{OH}$ lines to the temperature, the line appears much stronger in 3D than in 1D.

This effect is usually found in metal-poor atmospheres (see Asplund et al. 1999) and is particularly important for the oxygen abundances derived from $\mathrm{OH}$ molecules, as the differences are large in the layers where these lines are formed. The result is that the oxygen abundances become lower in the $3 \mathrm{D}$ formulation

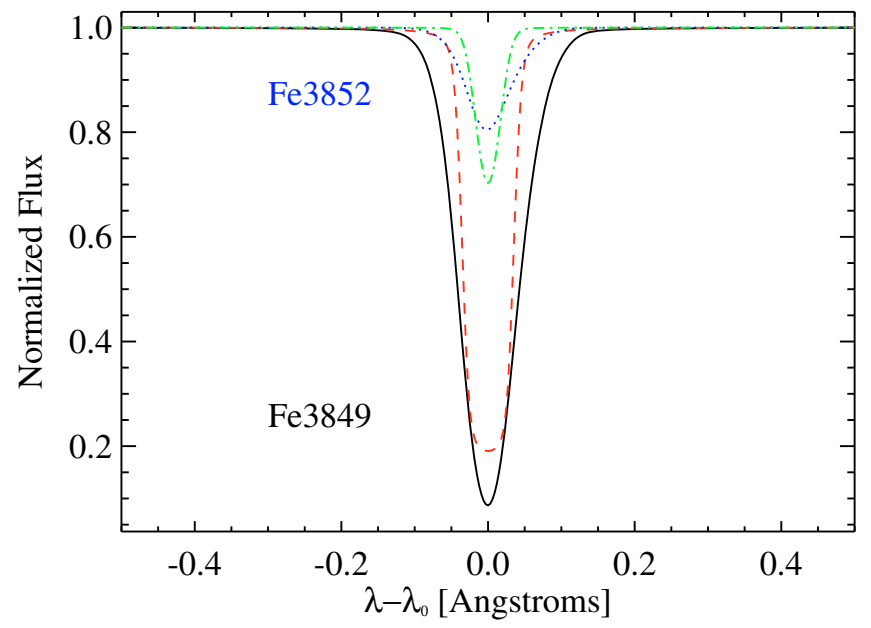

Fig. 5. Line profiles of the Fe I $3849 \AA$ and $3852 \AA$ lines computed with $\mathrm{CO}^{5} \mathrm{BOLD} 3 \mathrm{D}$ model (solid line for Fe I $3849 \AA$ And dotted line for Fe I $3852 \AA$ ) and $1 D_{\text {LHD }}$ model (dashed line for Fe I $3849 \AA$ and dasheddotted line for Fe I $3852 \AA$ ). The stellar parameters and metallicity of this simulation are $T_{\text {eff }}[\mathrm{K}] / \log \left(g\left[\mathrm{~cm} \mathrm{~s}^{-2}\right]\right) /[\mathrm{Fe} / \mathrm{H}]=6270 / 4 /-3$.

than in the $1 \mathrm{D}$. We quantify this effect through the $3 \mathrm{D}$ correction (see Sect. 2.3). In hotter 3D models $\left(T_{\text {eff }} \gtrsim 5900 \mathrm{~K}\right)$ this effect becomes more severe, making the $3 \mathrm{D}$ corrections larger.

We display the CFs to the $E W$ of the Fe I lines at $3849 \AA$ and $3852 \AA$ in Fig. 4. These lines have different excitation potentials ( $\chi=1.0$ and $2.2 \mathrm{eV}$ for the Fe $\mathrm{I} 3849 \AA$ and $3852 \AA$ lines) and their CFs are different in both strength and shape. The Fe I $3849 \AA$ line extends from $\log \tau \sim-4.5$ to almost 0 , whereas the Fe I $3852 \AA$ line, due to the higher excitation potential, goes from $\log \tau \sim-3.5$ to 0 . Nevertheless, the main contribution is located at $\log \tau \sim-3.5$ for the Fe I $3849 \AA$ line and at $\log \tau \sim-1$ for the Fe I $3852 \AA$ line. This means that the Fe I 3849 line is mostly formed in the outer layers of the $3 \mathrm{D}$ atmospheric model, and the equivalent width is larger than in the $1 \mathrm{D}_{\mathrm{LHD}}$ model. Although the Fe I $3852 \AA$ line is mainly formed in the inner layers, the $E W$ computed for this $3 \mathrm{D}$ model is still larger than in $1 \mathrm{D}$, due to the contribution of the outer layers. Therefore, this line still provides negative $3 \mathrm{D}$ corrections. In addition, although they have very similar excitation potentials, the ratio $E W(3 \mathrm{D}) / E W\left(1 \mathrm{D}_{\mathrm{LHD}}\right)$ of the Fe I $3849 \AA$ line is smaller than that of the $\mathrm{OH} 3167 \AA$ line. This is probably caused by the stronger sensitivity of the $\mathrm{OH}$ line to the temperature structure of the $3 \mathrm{D}$ model.

In Fig. 5 we display the line profiles of the Fe I $3849 \AA$ and $3852 \AA$ lines for the same $3 \mathrm{D}$ and $1 \mathrm{D}_{\mathrm{LHD}}$ models with stellar parameters and metallicity $T_{\text {eff }} / \log \left(g / \mathrm{cm} \mathrm{s}^{2}\right) /[\mathrm{Fe} / \mathrm{H}]=$ $6270 / 4 /-3$. The 3D profiles of both lines are more broadened than the $1 D_{\text {LHD }}$ profiles in part because these $1 \mathrm{D}$ profiles were not been convolved with a macroturbulent velocity, whereas in the $3 \mathrm{D}$ profiles the broadening effect due to macroturbulence is naturally included. In addition, the 3D profiles are slightly asymmetric due to the convective motions in the hydrodynamical simulations included in the 3D model atmosphere. It is quite clear that the $3 \mathrm{D}$ profile of the Fe I $3849 \AA$ line is stronger than the $1 D_{\text {LHD }}$ profile. This is not as evident for the Fe I $3852 \AA$ line, where the line core is deeper in the $1 \mathrm{D}_{\mathrm{LHD}}$ profile but the line wings are tighter. The ratios $E W(3 \mathrm{D}) / E W\left(1 \mathrm{D}_{\mathrm{LHD}}\right)$ of these lines in these $3 \mathrm{D}$ and $1 \mathrm{D}_{\mathrm{LHD}}$ models are 1.59 and 1.25 for the Fe I $3849 \AA$ and $3852 \AA$ lines, respectively. As a comparison, the ratios $E W(3 \mathrm{D}) / E W\left(1 \mathrm{D}_{\mathrm{LHD}}\right)$ of the $\mathrm{OH} 3123 \AA$ and $3167 \AA$ lines 
J. I. González Hernández et al.: Oxygen versus Iron using 3D models

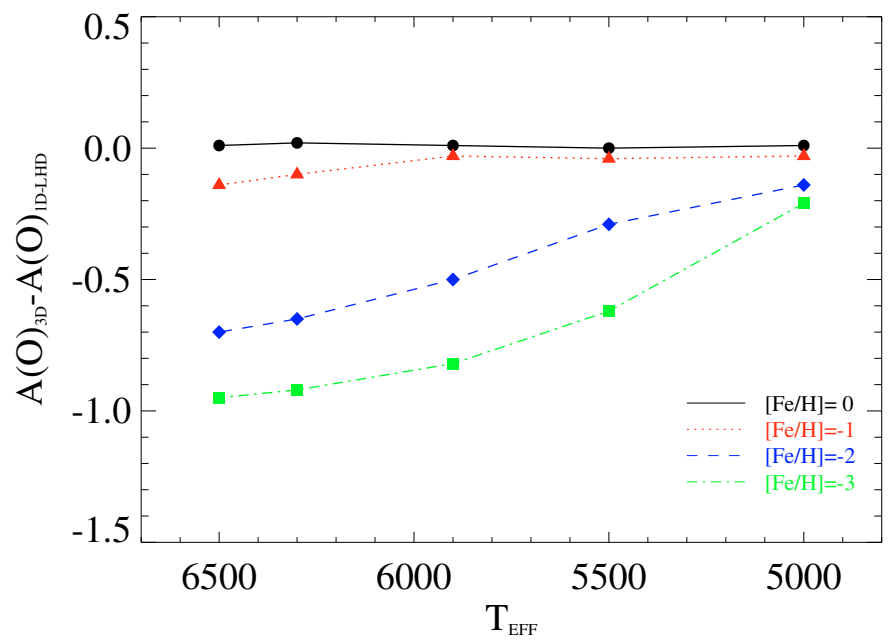

Fig. 6. 3D-1D $\mathrm{D}_{\mathrm{LHD}}$ corrections for different $T_{\text {eff }}$ and metallicities, computed for the $\mathrm{OH} 3167 \AA$ line. These 3D hydrodynamical model atmospheres have $\log g=4$.

are 20.2 and 8.2, respectively. Therefore, it is expected that all these lines should provide different $3 \mathrm{D}$ corrections. We will discuss quantitatively these abundance corrections in Sect. 2.3.

\section{3. $3 D$ corrections}

The spectral synthesis code Linfor3D produces three curves of growth: full 3D, $\langle 3 \mathrm{D}\rangle$ and $1 \mathrm{D}_{\mathrm{LHD}}$ (Caffau et al. 2009, 2010). This allows us to estimate abundance corrections through the $E W(3 \mathrm{D})$ of the $3 \mathrm{D}$ model abundance to derive the corresponding abundances in the $\langle 3 \mathrm{D}\rangle$ and $1 \mathrm{D}_{\mathrm{LHD}}$ models. Two main effects distinguish 3D from 1D models: the average temperature profile and the horizontal temperature fluctuations. We quantify the contribution of these two main effects by introducing the $3 \mathrm{D}$ correction as $3 \mathrm{D}-1 \mathrm{D}_{\mathrm{LHD}}$. The first and the second effect can also be separately estimated with the $\langle 3 \mathrm{D}\rangle-1 \mathrm{D}_{\mathrm{LHD}}$ and $3 \mathrm{D}-\langle 3 \mathrm{D}\rangle$ corrections, respectively. The velocity fluctuations (i.e. intrinsic velocity fluctuations and microturbulence) are other effects that also play a role, but their influence on the derived abundance, although not negligible, is not as relevant. In Fig. 6 we display the $3 \mathrm{D}-1 \mathrm{D}_{\mathrm{LHD}}$ abundance corrections of the $\mathrm{OH}$ $3167 \AA$ line for different effective temperatures and metallicities, and $\log g=4$. The $3 \mathrm{D}$ models with metallicities $[\mathrm{Fe} / \mathrm{H}]=-1$ and 0 show very small and mainly positive $3 \mathrm{D}-1 \mathrm{D}_{\mathrm{LHD}}$ corrections, whereas $3 \mathrm{D}$ models with $[\mathrm{Fe} / \mathrm{H}]=-3$ and -2 show strong, negative corrections. The $3 \mathrm{D}-1 \mathrm{D}_{\mathrm{LHD}}$ generally increases towards hotter 3D models and lower metallicites. We note here that the behaviour of other $\mathrm{OH}$ lines is practically the same although for slightly different values for the $3 \mathrm{D}-1 \mathrm{D}_{\mathrm{LHD}}$ abundance corrections.

In Figs. 7 and 8 we show the $3 \mathrm{D}-1 \mathrm{D}_{\mathrm{LHD}}$ abundance corrections of the Fe I $3849 \AA$ and $3852 \AA$ lines for different effective temperatures and metallicities, and $\log g=4$. The behaviour of the Fe I $3849 \AA$ line is quite similar to that of the $\mathrm{OH} 3167 \AA$ line, although with smaller $3 \mathrm{D}-1 \mathrm{D}_{\mathrm{LHD}}$ values. On the other hand, the Fe I $3852 \AA$ line behaves in a completely different way, and the $3 \mathrm{D}-1 \mathrm{D}_{\mathrm{LHD}}$ corrections depend on whether the line formation occurs mainly in the outer or inner region of the atmosphere of a given 3D model at a given temperature and metallicity.

In Fig. 9 we depict the $\langle 3 \mathrm{D}\rangle-1 \mathrm{D}_{\mathrm{LHD}}$ corrections of the OH $3167 \AA$ line for different effective temperatures and metallicities, and $\log g=4$. The $\langle 3 \mathrm{D}\rangle-1 \mathrm{D}_{\mathrm{LHD}}$ corrections reflect how

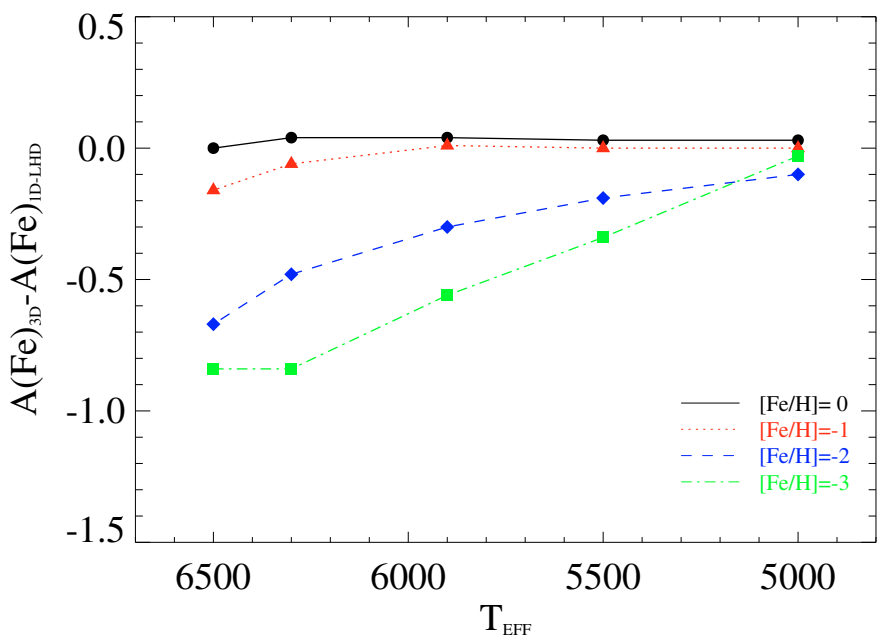

Fig. 7. Same as Fig. 6 but for the Fe I 3849 A.

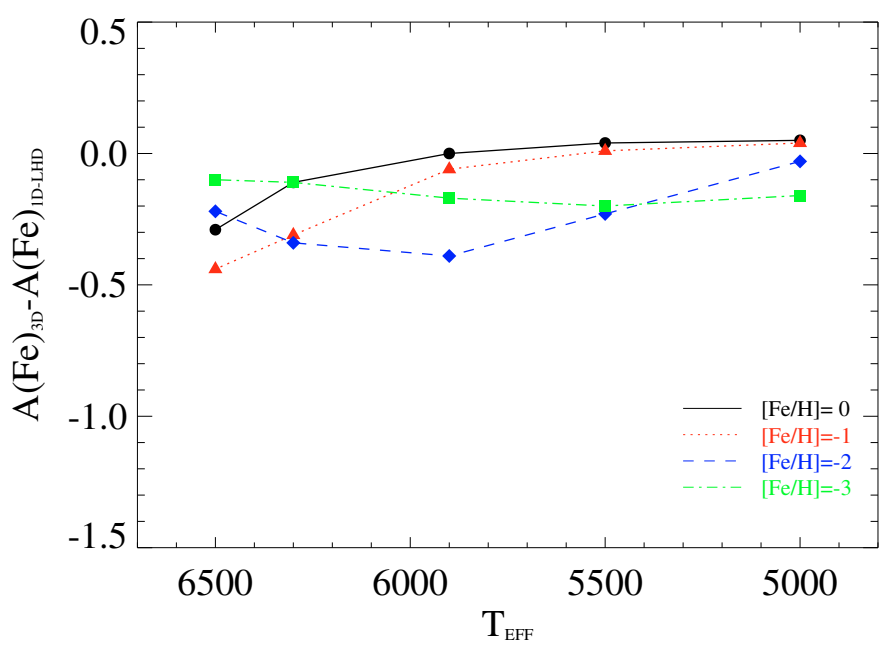

Fig. 8. Same as Fig. 6 but for the Fe I $3852 \AA$.

important these cooling effects are in the 3D models. Thus, the strongest correction is found for the 3D model with metallicity $[\mathrm{Fe} / \mathrm{H}]=-3$ and $T_{\text {eff }} \sim 5900$. One also realizes by comparing Figs. 6 and 9 that the 3D models with metallicities $[\mathrm{Fe} / \mathrm{H}]=-3$ and -2 and $T_{\text {eff }}>5900$ have large temperature fluctuations that account for a significant fraction of the total $3 \mathrm{D}-1 \mathrm{D}_{\mathrm{LHD}}$ correction.

The sensitivity of the $3 \mathrm{D}-1 \mathrm{D}_{\mathrm{LHD}}$ corrections to the surface gravity is less relevant (see Fig. 10). The derived $3 \mathrm{D}-1 \mathrm{D}_{\mathrm{LHD}}$ corrections are typically larger in 3D models with larger surface gravities, although only variations of $\lesssim 0.2$ dex are expected from $\log g=3.5$ to 4.5 .

The excitation potential determines the layer of the 3D model atmosphere where the line forms. Thus, it is expected that a line of the given molecule or atom forms further out if the excitation potential is lower. In the case of $\mathrm{OH}$ molecular lines, further out means lower temperature and therefore enhanced molecule formation. This is exactly what it is displayed in Fig. 11, the lower the excitation potential of a $\mathrm{OH}$ line is, the larger negative $3 \mathrm{D}-1 \mathrm{D}_{\mathrm{LHD}}$ corrections it provides.

All in all, the dependence of the abundance corrections on metallicity shows a kind of threshold behaviour. Noticeable corrections indicating sizable temperature differences between 1D and $3 \mathrm{D}$ models set in rather abruptly at metallicities below -1 . Ludwig et al. (2008) have argued that this can be expected on 


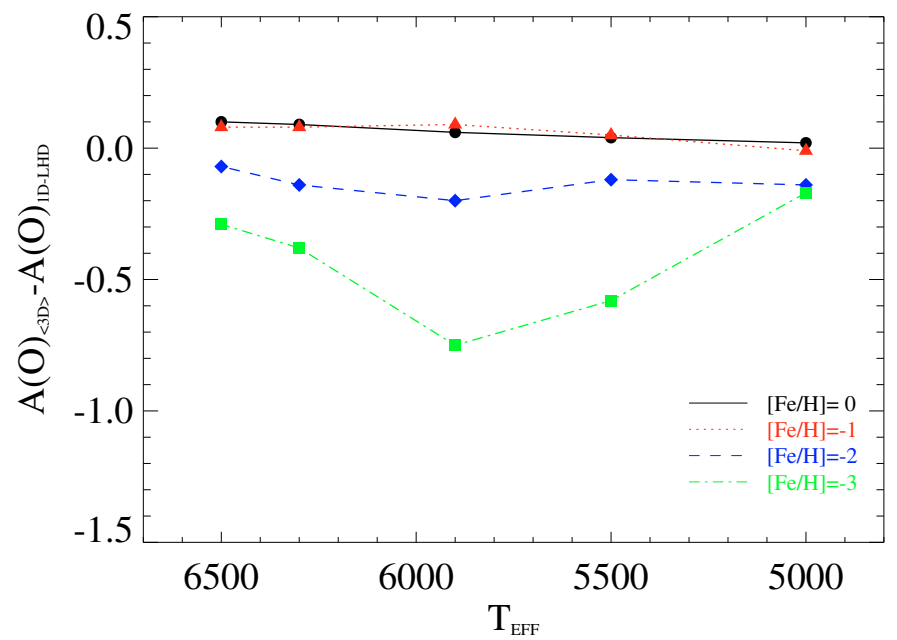

Fig. 9. $\langle 3 \mathrm{D}\rangle-1 \mathrm{D}_{\mathrm{LHD}}$ corrections for different $T_{\text {eff }}$ and metallicities, computed for the OH $3167 \AA$ line. These 3D hydrodynamical model atmospheres have $\log g=4$.

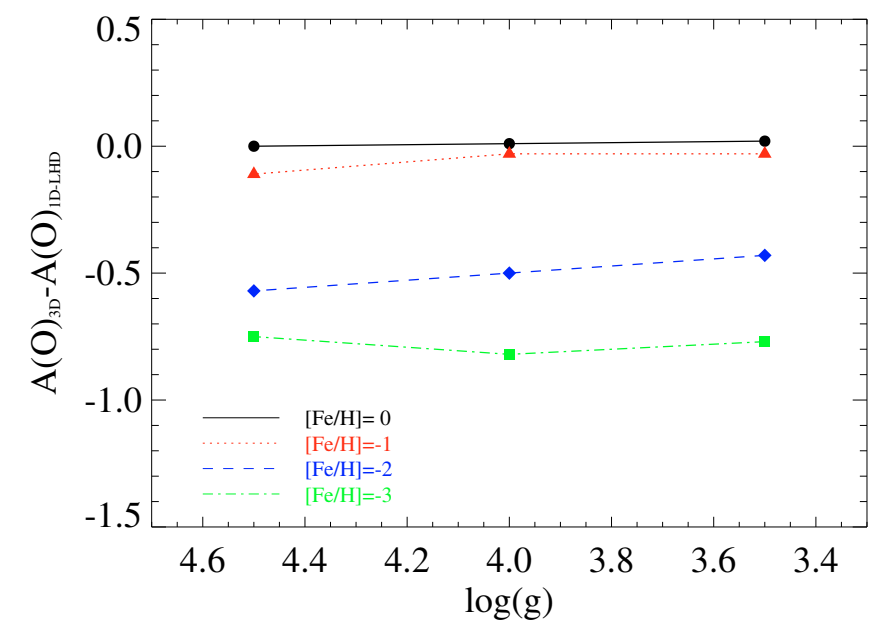

Fig. 10. 3D-1D $D_{\text {LHD }}$ corrections for different surface gravities and metallicities, computed for the $\mathrm{OH} 3167 \AA$ line. These 3D hydrodynamical model atmospheres have $T_{\text {eff }} \sim 5900 \mathrm{~K}$.

general grounds emerging from the temperature and metallicity sensitivity of the radiative relaxation time scale.

\subsection{Dependence on carbon abundance}

The molecular equilibrium of $\mathrm{OH}$ lines also involves the molecules $\mathrm{CO}, \mathrm{CH}$ and $\mathrm{C}_{2}$. Thus, the $\mathrm{C}$ abundance surely affects the contribution function to the $E W$ of the $\mathrm{OH}$ lines. The CO molecule is the most tightly bound and has the tendency to get hold of all available $\mathrm{O}$ atoms, to the detriment of $\mathrm{OH}$. Behara et al. (2010) have already checked this effect in $\mathrm{C}$-enhanced metal-poor stars, showing that if the $\mathrm{C}$ abundance is large enough, the $3 \mathrm{D}-1 \mathrm{D}_{\mathrm{LHD}}$ abundance corrections become much smaller, reaching values of the order of -0.2 if the carbonto-oxygen ratio is as high as $[\mathrm{C} / \mathrm{O}] \sim 1$. In Table 3 we provide the $3 \mathrm{D}-1 \mathrm{D}_{\text {LHD }}$ corrections to the $\mathrm{O}$ abundance derived from an $\mathrm{OH}$ line with excitation potential $\sim 0.1 \mathrm{eV}$, for several values of [C/O], which have been computed only for testing purposes with the $3 \mathrm{D}$ model with parameters $T_{\text {eff }}[\mathrm{K}] / \log \left(g\left[\mathrm{~cm} \mathrm{~s}^{-2}\right]\right) /[\mathrm{Fe} / \mathrm{H}]=$ $6270 / 4 /-3$. For these low ratios $[\mathrm{C} / \mathrm{O}]$, the $3 \mathrm{D}-1 \mathrm{D}_{\mathrm{LHD}}$ corrections are similar and within $0.15 \mathrm{dex}$. In addition, looking at these corrections in detail one realizes that (i) when $[\mathrm{C} / \mathrm{O}]$ is low

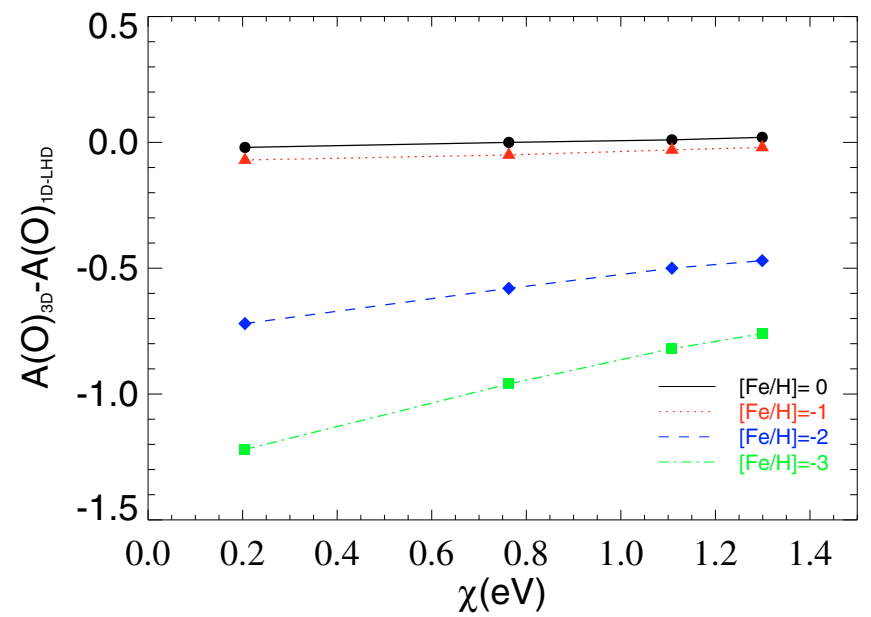

Fig. 11. 3D-1 $D_{\text {LHD }}$ corrections for different excitation potentials and metallicities, computed for $\mathrm{OH}$ lines. These 3D hydrodynamical models have $T_{\text {eff }} \sim 5900 \mathrm{~K}$ and $\log g=4$.

enough, the $3 \mathrm{D}-1 \mathrm{D}_{\text {LHD }}$ correction to the $\mathrm{O}$ abundance derived from $\mathrm{OH}$ lines is only slightly dependent on the $\mathrm{C}$ abundance with changes $\$ 0.05 \mathrm{dex}$; (ii) when the $[\mathrm{C} / \mathrm{O}]$ is near the value zero, the $\mathrm{C}$ abundance starts to become relevant, with changes $\sim 0.20$ dex; (iii) only when $[\mathrm{C} / \mathrm{O}]$ is large enough, the $3 \mathrm{D}-1 \mathrm{D}_{\mathrm{LHD}}$ correction becomes negligible.

In our computations we assumed that the carbon abundance scales with metallicity, $[\mathrm{C} / \mathrm{Fe}]=0$. In models with metallicities lower than -1 dex, we have assumed the $\alpha$-elements enhanced by a factor of $[\alpha / \mathrm{Fe}]=0.4$. Therefore, the $[\mathrm{C} / \mathrm{O}]=-0.4$, which means that at the lowest metallicities, i.e. at $[\mathrm{Fe} / \mathrm{H}]=-2$ and -3 , where the observed abundances can reach values of $[\mathrm{O} / \mathrm{Fe}] \sim 0.5-1.0$ (see Sect. 3) and $[\mathrm{C} / \mathrm{Fe}] \sim 0$ (e.g. Lai et al. 2007), our estimated 3D-1D $D_{\text {LHD }}$ corrections might have to be corrected downwards by $0.06-0.12$ dex. This small effect has not been taken into account and goes in the direction of slightly increasing the strength of the $3 \mathrm{D}-1 \mathrm{D}_{\mathrm{LHD}}$ corrections.

\subsection{Dependence on opacity binning}

We tested a few 3D hydrodynamical models with 12 opacity bins and found that the cooling effect at $\log \tau \lesssim-2$ is less pronounced than in our standard 6 bin models. From this result we expect that in the next generation of 3D models, using a refined opacity binning, the $3 \mathrm{D}-1 \mathrm{D}_{\mathrm{LHD}}$ abundance corrections would come out smaller for $\mathrm{OH}$ lines and $\mathrm{Fe}$ lines with excitation potentials below $1.5 \mathrm{eV}$. Therefore, the corrections presented here for these lines can be considered as upper limits.

In Table 2 we provide a comparison between the $3 \mathrm{D}-1 \mathrm{D}_{\mathrm{LHD}}$ corrections in the 6-bin and 12-bin 3D models. The 6-bin model produces larger corrections for these three $\mathrm{OH}$ lines, between $\sim-0.36$ and -0.23 dex, with excitation potentials between 0.2 and $1.1 \mathrm{eV}$. In addition, the 12-bin model has stronger temperature fluctuations than the 6-bin model, since the $3 \mathrm{D}-\langle 3 \mathrm{D}\rangle$ is larger for the 12-bin model. This difference explains some of the larger scatter in oxygen abundances obtained from different lines when using 3D models than in the 1D case (see Sect. 3).

\section{Applications to the Galactic oxygen abundance}

The aim of this study is to apply our grid of the $3 \mathrm{D}-1 \mathrm{D}_{\mathrm{LHD}}$ corrections to a sample of metal-poor dwarf stars with available $\mathrm{O}$ abundances derived from near-UV OH lines. This sample was 
Table 2. $3 \mathrm{D}$ abundance corrections for several $\mathrm{OH}$ lines using $3 \mathrm{D} \mathrm{CO}^{5} \mathrm{BOLD}$ models with 6 and 12 opacity bins with the parameters $T_{\text {eff }}[\mathrm{K}] / \log \left(g\left[\mathrm{~cm} \mathrm{~s}^{-2}\right]\right) /[\mathrm{Fe} / \mathrm{H}]=6270 / 4 /-3$ and $6240 / 4 /-3$, respectively.

\begin{tabular}{lrrrrrrr}
\hline \hline $\begin{array}{l}\text { Wavelength } \\
{[\AA]}\end{array}$ & $\begin{array}{r}\chi \\
{[\mathrm{eV}]}\end{array}$ & $\begin{array}{r}3 \mathrm{D}-\langle 3 \mathrm{D}\rangle_{, 6-\text { bin }} \\
{[\mathrm{dex}]}\end{array}$ & $\begin{array}{r}3 \mathrm{D}-\langle 3 \mathrm{D}\rangle_{, 12-\text { bin }} \\
{[\mathrm{dex}]}\end{array}$ & $\begin{array}{r}\Delta_{6-12 \mathrm{bin}} \\
{[\mathrm{dex}]}\end{array}$ & $\begin{array}{r}3 \mathrm{D}-1 \mathrm{D}_{\text {LHD }, 6-\text { bin }} \\
{[\mathrm{dex}]}\end{array}$ & $\begin{array}{r}3 \mathrm{D}-1 \mathrm{D}_{\text {LHD,12-bin }} \\
{[\mathrm{dex}]}\end{array}$ & $\begin{array}{r}\Delta_{6-12 \text { bin }} \\
{[\mathrm{dex}]}\end{array}$ \\
\hline 3128.287 & 0.210 & -0.852 & -0.953 & 0.101 & -1.416 & -1.057 & -0.359 \\
3139.170 & 0.763 & -0.647 & -0.735 & 0.088 & -1.083 & -0.806 & -0.277 \\
3167.169 & 1.108 & -0.544 & -0.622 & 0.078 & -0.910 & -0.675 & -0.235 \\
\hline
\end{tabular}

Table 3. $3 \mathrm{D}-1 \mathrm{D}_{\mathrm{LHD}} \mathrm{O}$ abundance corrections for several $[\mathrm{C} / \mathrm{O}]$ ratios from the $\mathrm{OH}$ line $3106 \AA$ with an excitation potential equal to $0.1 \mathrm{eV}$, using the 3D model with the parameters $T_{\text {eff }}[\mathrm{K}] / \log \left(g\left[\mathrm{~cm} \mathrm{~s}^{-2}\right]\right) /[\mathrm{Fe} / \mathrm{H}]=$ $6270 / 4 /-3$.

\begin{tabular}{lrrr}
\hline \hline$[\mathrm{C} / \mathrm{O}]$ & $\begin{array}{r}{[\mathrm{C} / \mathrm{Fe}]} \\
{[\mathrm{dex}]}\end{array}$ & $\begin{array}{r}{[\mathrm{O} / \mathrm{Fe}]} \\
{[\mathrm{dex}]}\end{array}$ & $\begin{array}{r}3 \mathrm{D}-1 \mathrm{D}_{\mathrm{LHD}} \\
{[\mathrm{dex}]}\end{array}$ \\
\hline-1.0 & 0.0 & 1.0 & -1.53 \\
-0.6 & 0.4 & 1.0 & -1.49 \\
-0.2 & 0.8 & 1.0 & -1.37 \\
\hline
\end{tabular}

obtained from the studies of Boesgaard et al. (1999), Israelian et al. $(1998,2001)$, and the most metal-poor dwarf stars of the binary CS 22876-032 from González Hernández et al. (2008). We adopted the stellar parameters and the $\mathrm{O}$ and $\mathrm{Fe} \mathrm{I}$ abundances of the dwarf stars provided in those works. In Fig. 13 we display the 1D-LTE $[\mathrm{O} / \mathrm{H}]$ and $1 \mathrm{D}-\mathrm{NLTE}[\mathrm{O} / \mathrm{Fe} \mathrm{I}]$ ratios versus $1 \mathrm{D}-\mathrm{NLTE}$ $[\mathrm{Fe} \mathrm{I} / \mathrm{H}]$ in metal-poor dwarf stars.

We applied the same formula given in Israelian et al. (2001) to correct the 1D Fe I abundances for NLTE. This formula was derived by these authors by fiting the 1D NLTE-LTE corrections provided by Thévenin \& Idiart (1999) as a function of metallicity. They also noted that these NLTE corrections were not significantly sensitive to the stellar parameters in their sample of metal-poor stars. This formula provides corrections of +0.25 and +0.37 at $[\mathrm{Fe} / \mathrm{H}] \sim-2$ and -4 , respectively. In the literature there are other studies of the NLTE effect on Fe lines (e.g. Korn et al. 2003; Shchukina et al. 2005; Mashonkina et al. 2010) in metal-poor stars, but none of them provides a similar table with NLTE-LTE corrections for a grid of models at different metallicities. NLTE effects on the $\mathrm{OH}$ lines may be also significant. Asplund \& García Pérez (2001) tried to investigate this effect using a two-level $\mathrm{OH}$ molecule and found NLTE corrections up to +0.25 dex at metallicities below -2 . We note here that in this study we have not taken into account any possible NLTE effect on the $\mathrm{OH}$ lines. The trend displayed in Fig. 13 clearly shows an increasing $[\mathrm{O} / \mathrm{Fe} \mathrm{I}]$ ratio towards lower metallicities. The error bars in this figure only show the dispersion of the abundance measurements using 1D models. We derived the slope of a linear fit to all data points shown in the lower panel in Fig. 13, providing $-0.39 \pm 0.03$. This value may be compared the slope of the 1D-NLTE [O/Fe] ratio reported in Israelian et al. (2001), whose value is $-0.33 \pm 0.02$. The difference is likely related to the fact that Israelian et al. (2001) used data points from Israelian et al. (1998, 2001), Boesgaard et al. (1999) and Edvardsson et al. (1993).

We determined for all 3D models in Table 1 the $3 \mathrm{D}-1 \mathrm{D}_{\mathrm{LHD}}$ abundance corrections for the $\mathrm{OH}$ and $\mathrm{Fe} I$ lines given in Table 4. The Fe I lines have three different excitation potentials from roughly 1 to $3 \mathrm{eV}$. We chose some stars in the sample of
Table 4. Selected lines for the abundance analysis.

\begin{tabular}{llrl}
\hline \hline $\begin{array}{l}\text { Wavelength } \\
{[\AA]}\end{array}$ & Transition & $\begin{array}{r}\chi \\
{[\mathrm{eV}]}\end{array}$ & $\log g f$ \\
\hline 3123.949 & OH $P_{11}(9.5)$ & 0.205 & -1.960 \\
3128.287 & OH $P_{22}(8.5)$ & 0.210 & -2.021 \\
3139.170 & OH $Q_{22}(17.5)$ & 0.763 & -1.559 \\
3167.169 & OH $Q R_{22}(21.5)$ & 1.108 & -1.541 \\
3255.493 & OH $P_{22}(23.5)$ & 1.299 & -1.809 \\
3843.257 & Fe I & 3.047 & -0.241 \\
3849.967 & Fe I & 1.011 & -0.871 \\
3852.573 & Fe I & 2.176 & -1.185 \\
\hline
\end{tabular}

Israelian et al. (1998, 2001), for which we have near-UV spectra to check if the selected Fe I lines provide appropriate $[\mathrm{Fe} / \mathrm{H}]$ values. For this estimate we used the code 1D LTE MOOG (Sneden 1973) and LTE model atmospheres with $\alpha$-elements enhanced, by +0.4 dex, computed with the Linux version (Sbordone et al. 2004) of the ATLAS code (Kurucz 1993). We used the new opacity distribution functions (ODFs) of Castelli \& Kurucz (2003) with the corresponding metallicity. In Table 5 we give the 1D LTE Fe abundances from these Fe I lines. The Fe I $3849 \AA$ line is quite strong and starts to be in the saturation regime for metallicities above -2 . If one takes into account that the 3D-LTE corrections for Fe I lines of different excitation potentials are different, one should obtain different abundances when using 1D models, according to Fig. 11. We hardly see this strong effect in the values given in Table 5. In Fig. 12 we depict these Fe I abundances for different values of microturbulent velocities for three of the stars in Table 5. As soon as one goes to higher metallicities and lower excitation potentials, the Fe I is more sensitive to the adopted microturbulence. However, at the lowest metallicities, the Fe I line at $3 \mathrm{eV}$ provides more uncertain results because the $E W$ is very low and therefore more sensitive to the quality of the observed spectra. We believe that our choice of microturbulent velocity equal to $1 \mathrm{~km} \mathrm{~s}^{-1}$ is a reasonable assumption, although the hottest stars, with $T_{\text {eff }} \sim 6300 \mathrm{~K}$, in our sample may require a slightly higher microturbulence.

In order to derive $3 \mathrm{D}$ abundances, we interpolated within the grid of 3D-1D $\mathrm{D}_{\mathrm{LHD}}$ corrections using the stellar parameters and metallicities of the sample stars. For $\mathrm{OH}$ lines, we applied individual abundance $3 \mathrm{D}-1 \mathrm{D}_{\mathrm{LHD}}$ corrections to the $\mathrm{O}$ abundance derived from each $\mathrm{OH}$ line and finally computed the average 3D O abundance of all available lines in each star. The error of the $[\mathrm{O} / \mathrm{H}]$ abundance is then estimated from the dispersion of the 3D-LTE abundances of all $\mathrm{OH}$ lines.

For Fe I we used a different recipe. The papers by Israelian et al. (1998) and Boesgaard et al. (1999) do not provide the Fe I lines used to determine the metallicity. On the other hand, 


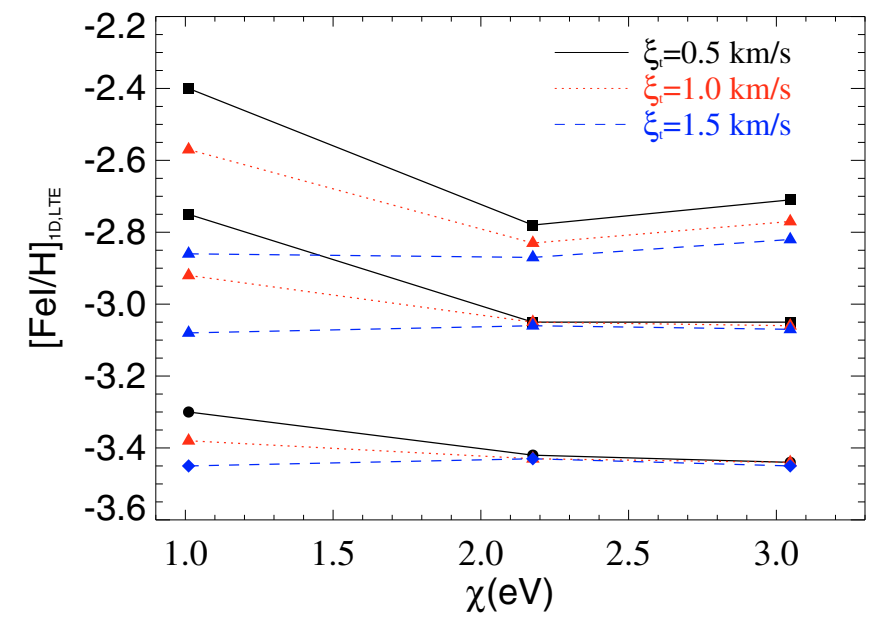

Fig. 12. 1D LTE $[\mathrm{Fe} / \mathrm{H}]$ abundances of some the stars in Table 5 excitation potential of the $\mathrm{Fe} I$ line, for three different microturbulent velocities adopted in each computation.

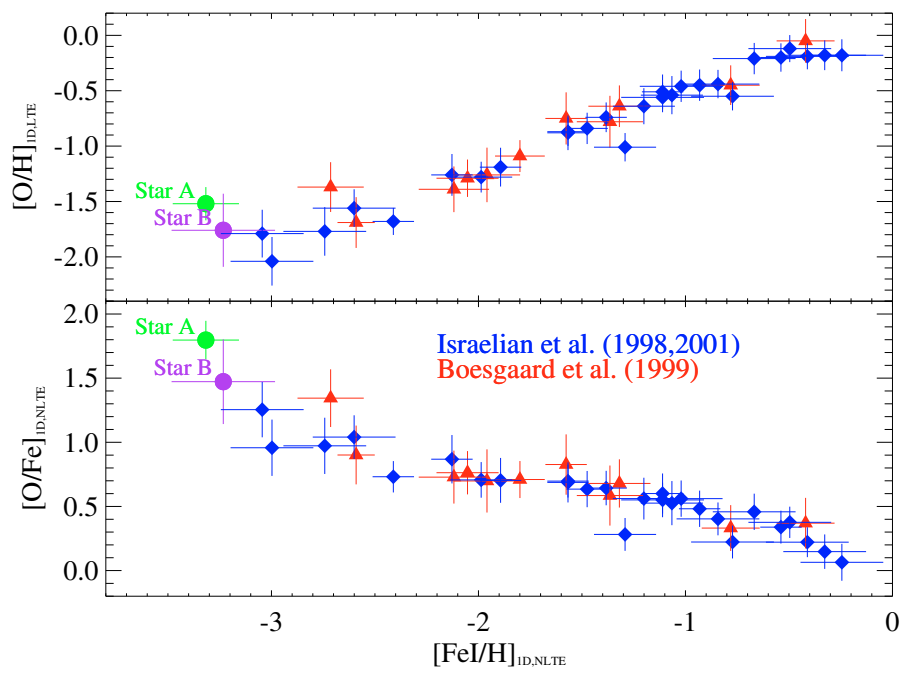

Fig. 13. 1D $[\mathrm{O} / \mathrm{Fe} \mathrm{I}]$ ratio versus $1 \mathrm{D}$ Fe I abundances in metal-poor dwarf stars computed with 1D-LTE models using $\mathrm{OH}$ lines from Boesgaard et al. (1999, triangles), Israelian et al. (1998, 2001, diamonds). The circles represent the most metal-poor dwarf stars of the binary CS 22876-032 from González Hernández et al. (2008). The stars labeled "A" and "B" represent the primary and secondary star in this binary.

the paper by Israelian et al. (2001) gives a few Fe I lines in the near-UV spectral region where the $\mathrm{OH}$ lines form. The excitation potential of these Fe I lines is $\chi \sim 1 \mathrm{eV}$. However, these Fe I line are too strong and gets saturated in stars with $[\mathrm{Fe} / \mathrm{H}]>-2$. Therefore, we decided to adopt the following strategy: (i) for stars with $[\mathrm{Fe} / \mathrm{H}]<-2$ we only used the Fe I $3849 \AA$ line to apply a $3 \mathrm{D}-1 \mathrm{D}_{\mathrm{LHD}}$ correction to the $1 \mathrm{D}$ metallicities; and (ii) for stars with $[\mathrm{Fe} / \mathrm{H}]>-2$ we derived an average correction with the two other Fe I lines at 3843 and $3852 \AA$. For $[\mathrm{Fe} / \mathrm{H}]>-2$, we estimated the error of the 3D-LTE $[\mathrm{Fe} \mathrm{I} / \mathrm{H}]$ by adding quadratically the dispersion of the 3D-1 $\mathrm{D}_{\mathrm{LHD}}$ corrections and the dispersion of the 1D-LTE abundances. For $[\mathrm{Fe} / \mathrm{H}]<-2$, we adopted ad hoc a dispersion of 0.3 dex in the $3 \mathrm{D}-1 \mathrm{D}_{\mathrm{LHD}}$ abundance corrections because we were only using one Fe I line. In Sect. 2.3 we discussed the expected differences in the 3D-LTE 3D-1D $\mathrm{D}_{\text {LHD }}$ correction between the Fe I $3849 \AA$ and $3852 \AA$ lines for models with $[\mathrm{Fe} / \mathrm{H}]<-2$. Thus, it is clear that if one would use both

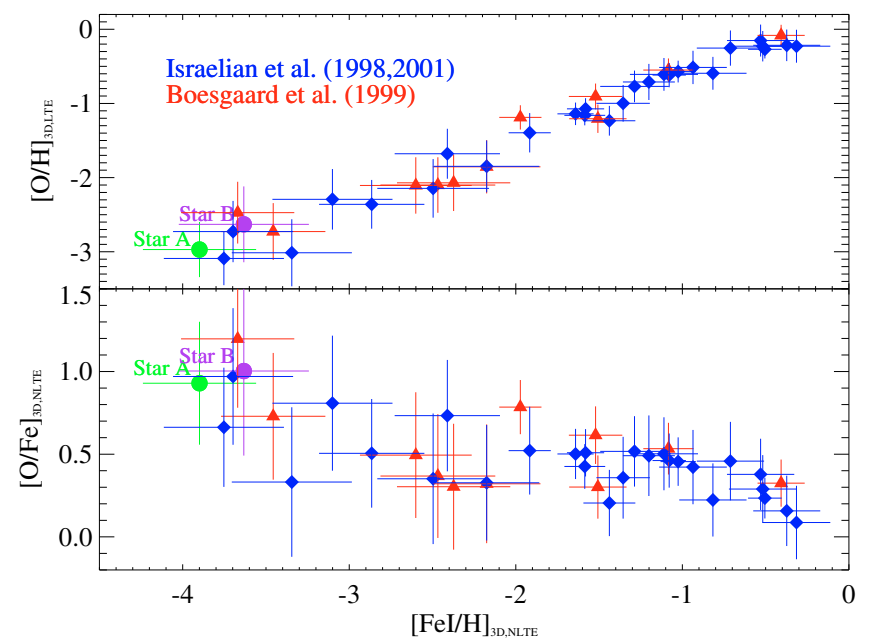

Fig. 14. 3D-LTE $[\mathrm{O} / \mathrm{H}]$ and $3 \mathrm{D}-\mathrm{NLTE}[\mathrm{O} / \mathrm{Fe} \mathrm{I}]$ ratios versus $3 \mathrm{D}-\mathrm{NLTE}$ $\mathrm{Fe}$ I abundances computed with 3D-LTE models. The symbols are the same as Fig. 13.

Fe I lines to estimate the 3D-1D $\mathrm{D}_{\mathrm{LHD}}$ correction for stars with $[\mathrm{Fe} / \mathrm{H}]<-2$, one would get smaller corrections, but the error bar associated to these corrections would be very large.

As a first approximation, we corrected our values for the 3D-LTE Fe I abundances for NLTE effects with the 1D-NLTE corrections already applied before to the 1D case. Finally, we determined the 3D-NLTE [O/Fe I] ratio by computing the difference between the 3D-LTE O abundance and the 3D-NLTE Fe I abundance in all dwarf stars of the sample. We are aware that these NLTE corrections may be too small, but we also know that our 3D-LTE corrections in both $\mathrm{OH}$ and Fe lines may be also overestimated because of the opacity binning used to compute our grid of 3D models (see Sect. 2.5). In addition, the carbon abundance may be another factor to take into account (see Sect. 2.4). We see that these effects tend to go in opposite directions when trying to define the slope, if it exists, of the ratio $[\mathrm{O} / \mathrm{Fe}]$ versus $[\mathrm{Fe} / \mathrm{H}]$ at the lowest metallicities.

In Fig. 14 we depict the 3D-LTE $[\mathrm{O} / \mathrm{H}]$ and 3D-NLTE $[\mathrm{O} / \mathrm{Fe} \mathrm{I}]$ trend in metal-poor dwarf stars. The slope of the linear fit to the 3D-NLTE $[\mathrm{O} / \mathrm{Fe} \mathrm{I}]$ trend is smaller in absolute value, $-0.16 \pm 0.04$, but still negative and relatively significant, at least under the assumptions made in this work. The error bars are larger in this figure than in Fig. 13 because we added quadratically the standard deviation on the $\mathrm{OH}$ and $\mathrm{Fe}$ abundances, and the dispersion on the $3 \mathrm{D}-1 \mathrm{D}_{\mathrm{LHD}}$ abundance corrections from different $\mathrm{OH}$ and $\mathrm{Fe}$ lines.

There is also a larger scatter in the almost linear relation between the 3D-NLTE $[\mathrm{O} / \mathrm{Fe}]$ and $[\mathrm{Fe} / \mathrm{H}]$ ratios. The reason may be that the 3D corrections applied to the stars depend not only on the metallicity of the star, but also on the stellar parameters. Therefore, when we apply the $3 \mathrm{D}-1 \mathrm{D}_{\mathrm{LHD}}$ corrections, different stars move to different directions in the plane $[\mathrm{O} / \mathrm{Fe}]$ versus $[\mathrm{Fe} / \mathrm{H}]$. As we stated in Sect. 1, large and positive 3D-NLTE corrections for $\mathrm{Fe}$ I lines are expected in metal-poor 3D models (Shchukina et al. 2005), but this needs to be done for the whole grid of 3D hydrodynamical models to see how the picture changes. We also note here that the computations done by Shchukina et al. (2005) do not consider the inelastic collisions with neutral hydrogen atoms, because there is at present no reliable values for the collisional rates, and the classical Drawin formula leads to uncertain estimates. They also pointed out that inelastic collisions with neutral hydrogen atoms would tend to 
Table 5. 1D Fe abundances for six stars in the sample of Israelian et al. (1998, 2001), from three Fe I lines of different excitation potentials.

\begin{tabular}{lrrrrrrr}
\hline \hline Star & $\begin{array}{c}T_{\text {eff }} \\
{[\mathrm{K}]}\end{array}$ & $\begin{array}{c}\log g \\
{[\mathrm{dex}]}\end{array}$ & $\begin{array}{r}\xi_{t} \\
{\left[\mathrm{~km} \mathrm{~s}^{-1}\right]}\end{array}$ & $\begin{array}{r}{[\mathrm{Fe} / \mathrm{H}]} \\
{[\mathrm{dex}]}\end{array}$ & $\begin{array}{r}{[\mathrm{Fe} \mathrm{I} / \mathrm{H}]_{3143}} \\
{[\mathrm{dex}]}\end{array}$ & $\begin{array}{r}{[\mathrm{Fe} \mathrm{I} / \mathrm{H}]_{3149}} \\
{[\mathrm{dex}]}\end{array}$ & $\begin{array}{r}{[\mathrm{Fe} \mathrm{I} / \mathrm{H}]_{3152}} \\
{[\mathrm{dex}]}\end{array}$ \\
\hline G 64-12 & 6318 & 4.20 & 1.0 & -3.37 & -3.44 & -3.38 & -3.43 \\
G 64-37 & 6310 & 4.20 & 1.0 & -3.22 & -3.22 & -3.21 & -3.19 \\
LP 815-43 & 6265 & 4.54 & 1.0 & -3.05 & -3.06 & -2.92 & -3.05 \\
HD 140283 & 5550 & 3.35 & 1.0 & -2.70 & -2.77 & -2.57 & -2.83 \\
HD 166913 & 5965 & 3.90 & 1.0 & -1.80 & - & -1.57 & -1.78 \\
HD 76932 & 5800 & 3.85 & 1.0 & -1.10 & - & -1.48 & -1.45 \\
\hline
\end{tabular}

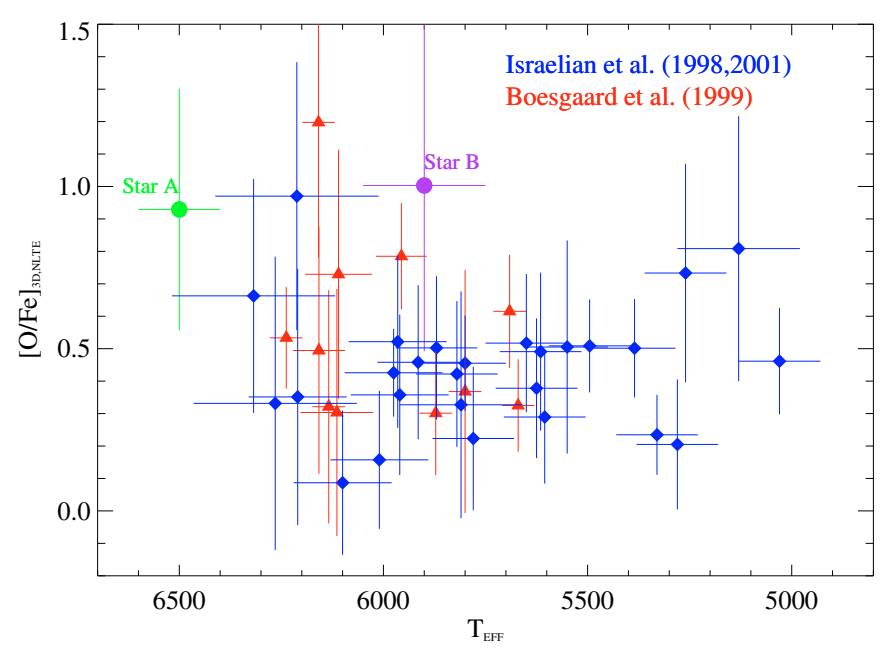

Fig. 15. 3D $[\mathrm{O} / \mathrm{Fe} \mathrm{I}]$ ratio versus effective temperature. The symbols are the same as Fig. 13.

alleviate the NLTE effects, and therefore, the difference between the NLTE and LTE may be considered as the maximum effect that inelastic collisions might produce. Figure 15 shows the $3 \mathrm{D}-\mathrm{NLTE}[\mathrm{O} / \mathrm{Fe} \mathrm{I}]$ ratio versus effective temperature of the star. There is no clear trend with effective temperature, except that due to selection effects, most of the dwarf stars with higher $3 \mathrm{D}-\mathrm{NLTE}[\mathrm{O} / \mathrm{Fe} \mathrm{I}]$ values are hot stars $\left(T_{\text {eff }}>5900\right)$, with the exception of very few cases.

Finally, we remark here that after all these corrections, the trend in $[\mathrm{O} / \mathrm{Fe} \mathrm{I}]$ obtained from near-UV OH lines in metal-poor dwarf stars is consistent with $[\mathrm{O} / \mathrm{Fe}]$ ratios obtained from the forbidden $[\mathrm{OI}]$ in metal-poor giant stars for which the $3 \mathrm{D}$ corrections are expected to be negligible (González Hernández et al. 2008).

\section{Summary}

The large grid of 3D hydrodynamical model atmospheres of dwarf stars quite captivated our attention during the last three years (Ludwig et al. 2009). It took thousands of hours in computing time to build such a grid. We have used 52 3D models extracted from this grid with the following stellar parameters and metallicities: $T_{\text {eff }}=5000,5500,5900,6300$ and $6500 \mathrm{~K}$, $\log g=3.5,4$ and 4.5 , and $[\mathrm{Fe} / \mathrm{H}]=0,-1,-2$ and -3 . The main difference with the "classical" $1 \mathrm{D}$ models is that 3D models show temperature inhomogeneities and a cooler average temperature profile in models with $[\mathrm{Fe} / \mathrm{H}]<-1$.

This allowed us for the first time to compute 3D abundance corrections of several near-UV OH and Fe I lines. These 3D corrections are generally larger for higher effective temperatures, larger surface gravities, and lower metallicities. In addition, lines with lower excitation potentials show stronger 3D corrections.

We applied this grid of 3D corrections to a sample of metalpoor dwarf stars from Israelian et al. (1998, 2001), Boesgaard et al. (1999) and the most metal-poor dwarf stars of the binary CS 22876-032 from González Hernández et al. (2008). We interpolated within this grid, using the stellar parameters and metallicities of these stars.

Finally, we are able to display a new trend with the 3D-NLTE $[\mathrm{O} / \mathrm{Fe} \mathrm{I}]$ ratio versus metallicity and this trend still increases towards lower metallicities, as the 1D-NLTE, but with a smaller slope in absolute value. However, we caution that some assumptions (as e.g. the restriction to a 6-bin scheme in most 3D models and NLTE corrections only in 1D), which we believe to be reasonable, have been adopted to achieve this result and that therefore, this result should be taken with that in mind. A full 3D-NLTE study of all 3D model atmospheres presented in this paper must be performed for both near-UV OH lines and $\mathrm{Fe}$ I lines to see if this trend changes.

Acknowledgements. J.I.G.H., P.B., H.-G.L. and N.B. acknowledge support from the EU contract MEXT-CT-2004-014265 (CIFIST). J.I.G.H. also thanks support from the project AYA2008-00695 of the Spanish Ministry of Education and Science. B.F. acknowledges financial support from the Agence Nationale de la Recherche (ANR), and the "Programme National de Physique Stellaire" (PNPS) of CNRS/INSU, France. We are also grateful to the supercomputing centre CINECA, which has granted us time to compute part of the hydrodynamical models used in this investigation, through the INAF-CINECA agreement 2006, 2007.

\section{References}

Abia, C., \& Rebolo, R. 1989, ApJ, 347, 186

Asplund, M., \& García Pérez, A. E. 2001, A\&A, 372, 601

Asplund, M., Gustafsson, B., Kiselman, D., \& Eriksson, K. 1997, A\&A, 318, 521

Asplund, M., Nordlund, A., Trampedach, R., \& Stein, R. F. 1999, A\&A, 346, L17

Barbuy, B. 1988, A\&A, 191, 121

Behara, N. T., Bonifacio, P., Ludwig, H. G., et al. 2010, A\&A, 513, A72

Boesgaard, A. M., King, J. R., Deliyannis, C. P., \& Vogt, S. S. 1999, AJ, 117, 492

Caffau, E., Faraggiana, R., Bonifacio, P., Ludwig, H.-G., \& Steffen, M. 2007, A\&A, 470, 699

Caffau, E., Ludwig, H.-G., Steffen, M., et al. 2008, A\&A, 488, 1031

Caffau, E., Ludwig, H.-G., \& Steffen, M. 2009, Mem. Soc. Astron. Ital., 80, 643

Caffau, E., Ludwig, H.-G., Steffen, M., Freytag, B., \& Bonifacio, P. 2010, Sol. Phys., accepted [arXiv: 1003.1190]

Castelli, F., \& Kurucz, R. L. 2003, Modelling of Stellar Atmospheres, 210, 20

Cayrel, R., Depagne, E., Spite, M., et al. 2004, A\&A, 416, 1117

Edvardsson, B., Andersen, J., Gustafsson, B., et al. 1993, A\&A, 275, 101

Freytag, B., Steffen, M., \& Dorch, B. 2002, Astron. Nachri., 323, 213

Fulbright, J. P., \& Johnson, J. A. 2003, ApJ, 595, 1154 
García Pérez, A. E., Asplund, M., Primas, F., Nissen, P. E., \& Gustafsson, B. 2006, A\&A, 451, 621

González Hernández, J. I., Bonifacio, P., Ludwig, H.-G., et al. 2008, A\&A, 480, 233

Israelian, G., García López, R. J., \& Rebolo, R. 1998, ApJ, 507, 805

Israelian, G., Rebolo, R., García López, R. J., et al. 2001, ApJ, 551, 833

Kiselman, D. 2001, New Astron. Rev., 45, 559

Korn, A. J., Shi, J., \& Gehren, T. 2003, A\&A, 407, 691

Kurucz, R. L. 1993, ATLAS9 Stellar Atmospheres Programs and $2 \mathrm{~km} \mathrm{~s}^{-1}$ Grid, CD-ROM No. 13, Smithsonian Astrophysical Observatory, Cambridge, MA, USA

Lai, D. K., Johnson, J. A., Bolte, M., \& Lucatello, S. 2007, ApJ, 667, 1185

Ludwig, H.-G. 1992, PhDT, University of Kiel

Ludwig, H.-G., Jordan, S., \& Steffen, M. 1994, A\&A, 284, 105

Ludwig, H.-G., González Hernández, J. I., Behara, N., Caffau, E., \& Steffen, M. 2008, First Stars III, 990, 268
Ludwig, H.-G., Caffau, E., Steffen, M., et al. 2009, Mem. Soc. Astron. It., 80, 711

Mashonkina, L., Gehren, T., Shi, J., Korn, A., \& Grupp, F. 2010, IAU Symp., 265,197

Nordlund, A. 1982, A\&A, 107, 1

Nissen, P. E., Primas, F., Asplund, M., \& Lambert, D. L. 2002, A\&A, 390, 235

Sbordone, L., Bonifacio, P., Castelli, F., \& Kurucz, R. L. 2004, Mem. Soc. Astron. Ital. Suppl., 5, 93

Shchukina, N. G., Trujillo Bueno, J., \& Asplund, M. 2005, ApJ, 618, 939

Sneden, C. 1973, Ph.D. Dissertation, Univ. of Texas, Austin

Steffen, M., Ludwig, H.-G., \& Freytag, B. 1995, A\&A, 300, 473

Stein, R. F., \& Nordlund, A. 1998, ApJ, 499, 914

Thévenin, F., \& Idiart, T. P. 1999, ApJ, 521, 753

Vögler, A., Bruls, J. H. M. J., \& Schüssler, M. 2004, A\&A, 421, 741

Wedemeyer, S., Freytag, B., Steffen, M., Ludwig, H.-G., \& Holweger, H. 2004, A\&A, 414, 1121 